\title{
Tunable lifetime multiplexing using luminescent
} nanocrystals

\author{
Yiqing Lu1, Jiangbo Zhao1, Run Zhang1,3, Yujia Liu1,4,5, Deming Liu1, Ewa M. Goldys', Xusan Yang4, \\ Peng $\mathrm{Xi}^{4}$, Anwar Sunna ${ }^{3}$, Jie Lu1,3, Yu Shi ${ }^{1,3}$, Robert C. Leif ${ }^{6}$, Yujing Huo ${ }^{7}$, Jian Shen ${ }^{8}$, James A. Piper ${ }^{1}$, \\ J. Paul Robinson ${ }^{2}$ and Dayong Jin ${ }^{1,2,3 \star}$
}

Optical multiplexing plays an important role in applications such as optical data storage ${ }^{1}$, document security ${ }^{2}$, molecular probes $^{3,4}$ and bead assays for personalized medicine ${ }^{5}$. Conventional fluorescent colour coding is limited by spectral overlap and background interference, restricting the number of distinguishable identities. Here we show that tunable luminescent lifetimes $\tau$ in the microsecond region can be exploited to code individual upconversion nanocrystals. In a single colour band, one can generate more than ten nanocrystal populations with distinct lifetimes ranging from $25.6 \mu \mathrm{s}$ to $662.4 \mu \mathrm{s}$ and decode their well-separated lifetime identities, which are independent of either colour or intensity. Such ' $\tau$-Dots' potentially suit multichannel bioimaging, high-throughput cytometry quantification, high-density data storage, as well as security codes to combat counterfeiting. This demonstration extends the optical multiplexing capability by adding the temporal dimension of luminescent signals, opening new opportunities in the life sciences, medicine and data security.

Multiplexing in biotechnology and life sciences refers to highthroughput technologies capable of simultaneous identification and quantification of multiple distinctive species ${ }^{6-15}$. Multiplexing molecular diagnostics impacts broadly on the bioinformatics areas of genomics ${ }^{16,17}$, proteomics ${ }^{18}$, metabolomics ${ }^{19}$, cytomics ${ }^{15}$ and personalized medicine ${ }^{5,20}$, where personal gene expression profiling holds promise for individually targeted therapies. In data storage, the main goal of multiplexing is to increase the data storage capacity within spatially limited memory elements ${ }^{1,21}$. In security printing of banknotes, identity cards, trademark tags and so on, it helps to prevent forgery, tampering or counterfeiting, and thermochromatic, magnetic, multicolour fluorescent and optically variable colourchanging inks have been used for this purpose ${ }^{2}$. Multiplexing typically requires a matrix of optical codes, ideally carried by nano-/micro-sized objects, each of which should be accurately identifiable at high speed and in a low-cost fashion.

Fluorescence colour codes used in a broad range of fields have been one of the most popular methods for multiplexing. As one example, cytometry combined with fluorescence staining has been at the frontier of rapid detection of single cells for the last several decades. However, the crowded spectral domain limits the modern multi-colour flow cytometry to fewer than twenty channels, requiring three to five lasers, tens of filters and up to twenty light detectors $^{22}$. The unavoidable spectral overlap requires complicated and tedious colour compensations. Thus, the outstanding challenge in multiplexing is to find ways of creating additional distinguishable coding dimensions, such as mass spectra ${ }^{15}$, fluorescence lifetime ${ }^{23}$ and Raman spectra ${ }^{24}$. These new dimensions become highly useful when the new identities can be accurately decoded (detected) by a simple, rapid and low-cost device.

In this Letter we report a new multiplexing concept by manipulating the luminescence decay lifetimes to create a temporal coding dimension in a wide microsecond-to-millisecond range. Among typical materials that emit such long-lived luminescence, rareearth doped luminescent nanocrystals are attracting significant attention with their applications in sensing, bioimaging, nanomedicine, solar cells and three-dimensional displays ${ }^{25}$. Recent advances in the synthesis of rare-earth doped upconversion nanocrystals, in particular, have led to accurate control of composite, crystal phase, morphology and emission colours ${ }^{26}$. Here, we have developed an approach to precisely tune the micro-/millisecond luminescent decays and produce individual populations of rare-earth doped nanocrystals with distinct lifetimes. Our results constitute a new optical coding and decoding dimension, which broadens the application of upconversion materials in nanoscale photonics.

Our lifetime multiplexing concept based on the tunability of lifetimes has been implemented following a simple scheme of co-doping sensitizer $\mathrm{Yb}^{3+}$ ions and blue-emitting $\mathrm{Tm}^{3+}$ ions at stepwise varied concentrations into the $\mathrm{NaYF}_{4}$ nanocrystals (Supplementary Section 1.1). In this case, energy transfer from the sensitizer to the emitter ion at varying sensitizer-emitter distances provides lifetime tunability (Fig. 1). By using this approach we have been able to tune the lifetime of $40 \mathrm{~nm} \mathrm{NaYF}_{4}: \mathrm{Yb}$,Tm nanocrystals in the blue emission band from $48 \mu \mathrm{s}(4 \mathrm{~mol} \% \mathrm{Tm})$ to $668 \mu \mathrm{s}(0.2 \mathrm{~mol} \% \mathrm{Tm})$. Thanks to the photostability of the nanocrystals and the background-free advantage of time-resolved detection, our purpose-built time-resolved confocal scanning system (Supplementary Sections 2.1 and 2.2) achieved a sufficiently high signal-to-background ratio (over $10^{2}$ ) for lifetime measurement of a single nanocrystal's emission at $475 \mathrm{~nm}$. Figure 1 shows the confocal images of five typical groups of lifetime-encoded Tm-doped ' $\tau$-Dots', using pseudocolour to map the luminescence lifetime for each pixel.

These $\tau$-Dots emit luminescence in the microsecond range, about three orders of magnitude longer than the background autofluorescence (less than $10 \mathrm{~ns}$ ), which can be easily suppressed through time-resolved measurements. We took advantage of this background-free readout for the rapid detection of single cells

\footnotetext{
'Advanced Cytometry Laboratories, MQ Photonics Research Centre and MQ BioFocus Research Centre, Macquarie University, Sydney, New South Wales 2109, Australia, ${ }^{2}$ Purdue University Cytometry Laboratories, Bindley Bioscience Center, Purdue University, West Lafayette, Indiana 47907, USA, ${ }^{3}$ Department of Chemistry and Biomolecular Sciences, Macquarie University, Sydney, New South Wales 2109, Australia, ${ }^{4}$ Department of Biomedical Engineering, College of Engineering, Peking University, Beijing 100871, China, ${ }^{5}$ School of Biomedical Engineering, Shanghai Jiao Tong University, Shanghai, China, ${ }^{6}$ Newport Instruments, 3345 Hopi Place, San Diego, California 92117-3516, USA, ${ }^{7}$ Department of Electronic Engineering, Tsinghua University, Beijing 100084, China, ${ }^{8}$ Olympus Australia, 82 Waterloo Road, North Ryde, New South Wales 2113, Australia. *e-mail: dayong.jin@mq.edu.au
} 

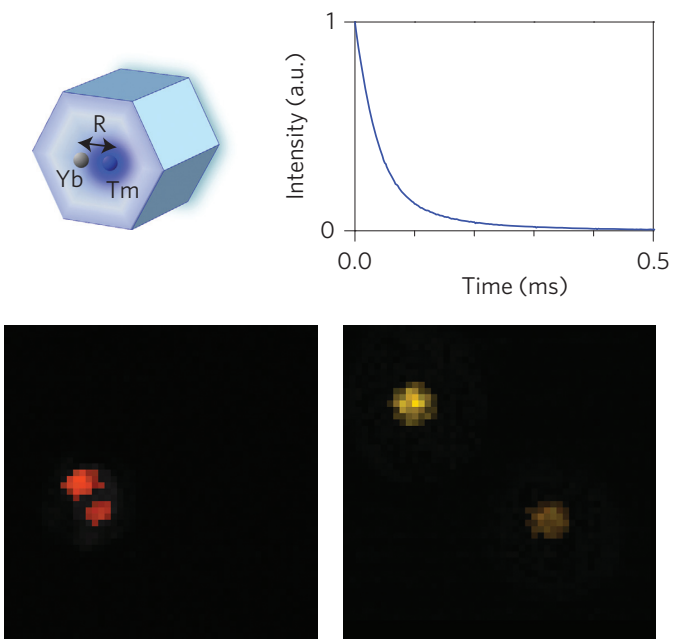

$126.5 \pm 9.9 \%$
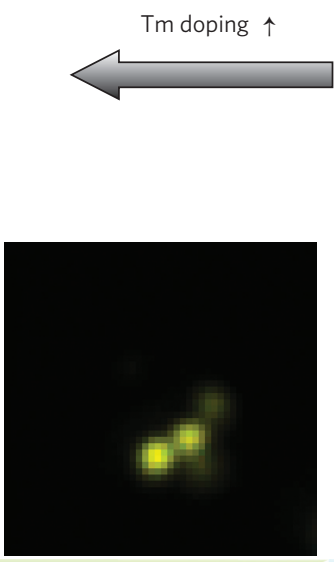

$182.6 \pm 6.6 \%$
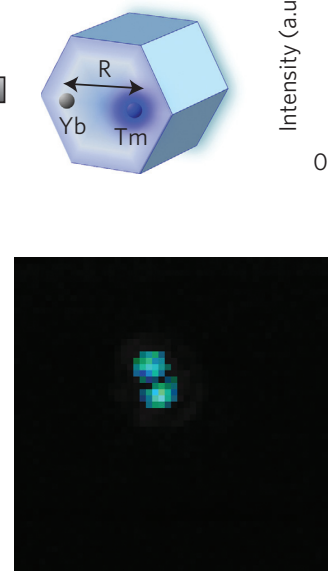

$501.1 \pm 8.8 \%$
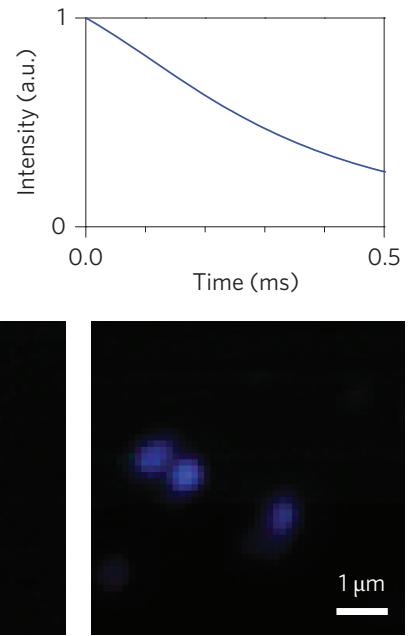

$668.8 \pm 5.1 \%$

Lifetime ( $\mu s$ )

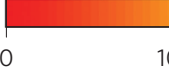

100

200

300

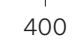

400

500

600

Figure 1 | Lifetime tuning scheme and time-resolved confocal images for $\mathrm{NaYF}_{4}: \mathbf{Y b}, \mathbf{T m}$ upconversion nanocrystals. Each pixel was excited for 200 $\mu$, followed by a delayed detection window of up to $3.8 \mathrm{~ms}$ to record its time-gated luminescence decay (40 ms exposure time to allow 10 times integration). The colour tone (hue) for each pixel represents its lifetime value decoded from the decay curve. The nanocrystals in the images from left to right have Tm doping concentrations of 4, 2, 1, 0.5 and 0.2 mol\%, respectively, as well as 20 mol\% Yb dopants.
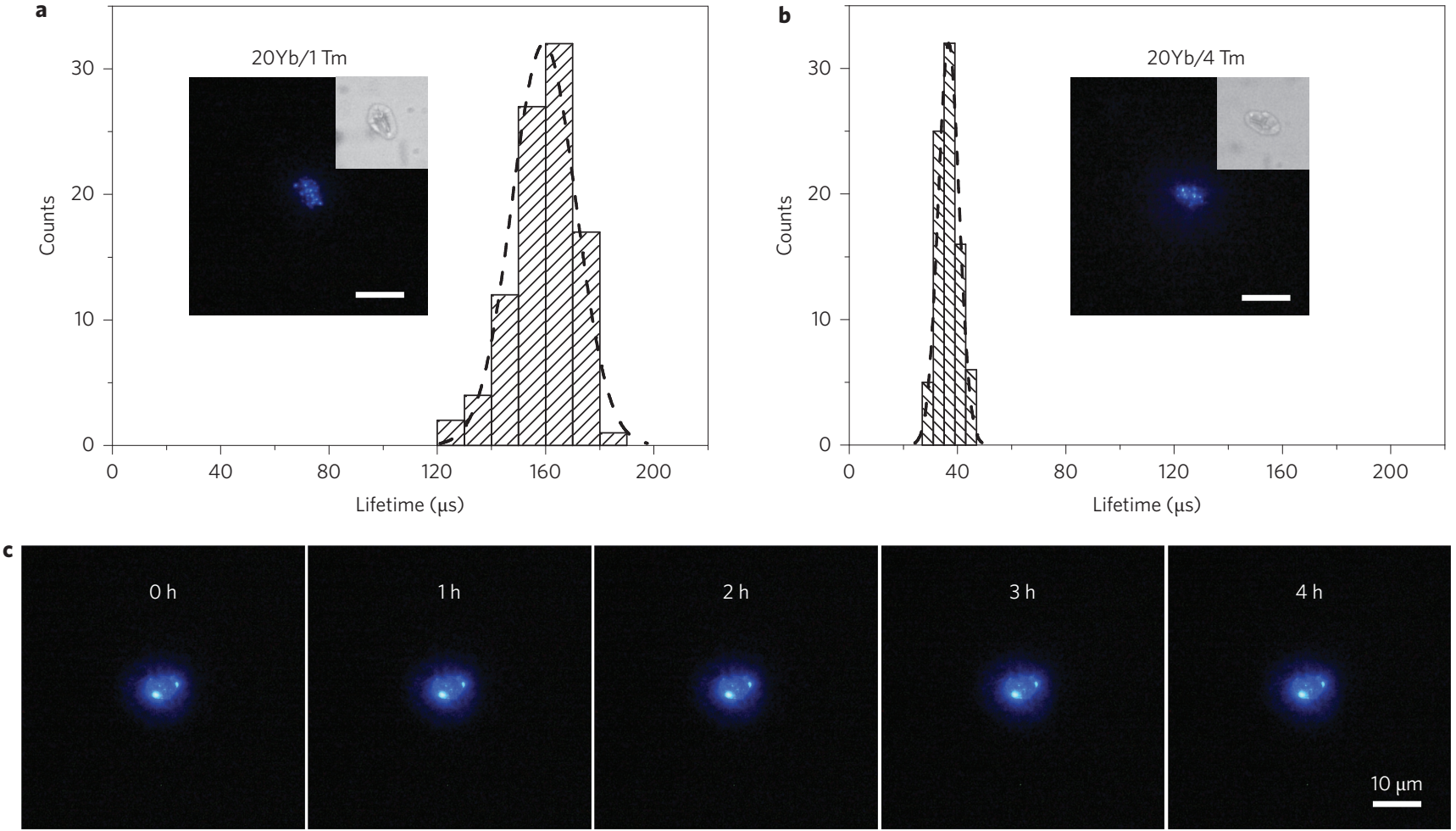

Figure 2 | Results for $\boldsymbol{\tau}$-Dots-labelled Giardia cysts measured by the time-resolved scanning cytometry system. a,b, Lifetime histograms obtained from cysts labelled with different lifetime-encoded $\tau$-Dots (Yb/Tm co-doping concentration (mol\%:mol\%) of 20:1 for $\mathbf{a}$ and 20:4 for $\mathbf{b}$ ). The scanning cytometry allows retrieval of each individual target cyst for luminescence as well as bright-field imaging confirmation. c, Typical recorded luminescence images for the same cyst under $4 \mathrm{~h}$ continuous laser excitation. All images were captured with a $100 \mathrm{~ms}$ exposure time.

with our time-gated orthogonal scanning automated microscopy (OSAM) platform ${ }^{27}$, which was further developed to a next-generation time-resolved scanning cytometry system (Supplementary
Section 2.3). The $\tau$-Dots with lifetime codes were surface-functionalized (Supplementary Sections 1.3 and 3), enabling bioconjugation to a number of different antibodies for simultaneously probing 
a

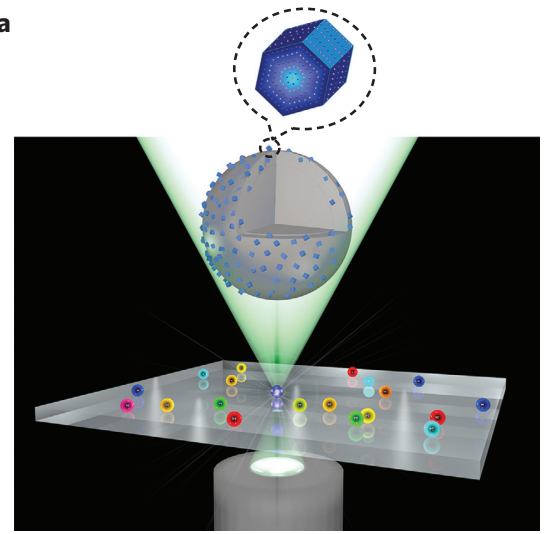

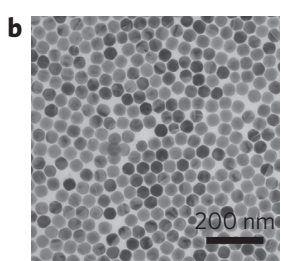

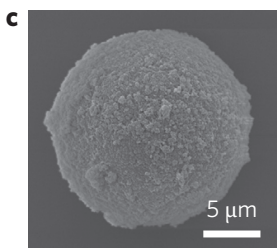

Figure 3 | Concept of $\boldsymbol{\tau}$-Dots-encoded microspheres as the lifetime multiplexing suspension arrays. a, The synthesized monodispersed Tm upconversion nanocrystals can be embedded into the shell of porous microspheres, which can be decoded by the time-resolved scanning cytometry system, for example. b,c, Typical TEM image of the nanocrystals (b) and SEM image of a microsphere incorporating the nanocrystals (c).

multiple rare-event pathogens (for example, Giardia lamblia, Escherichia coli O157:H7 and Cryptosporidium parvum) in beverages, food or within the hospital environment, in an effort to prevent disease or infection. Figure 2 presents a simple demonstration of two populations of $G$. lamblia cysts labelled with $1 \mathrm{~mol} \% \mathrm{Tm} \tau$-Dots with a lifetime of $\sim 160 \mu$ s and $4 \mathrm{~mol} \% \mathrm{Tm}$ $\tau$-Dots with a lifetime of $\sim 40 \mu$ s, respectively (Supplementary Section 4.1), which were rapidly scanned using the time-resolved

scanning cytometry system. The lifetime parameters yielded narrow population distributions (coefficients of variation $(\mathrm{CVs})<$ $10 \%$ ), offering superior detection accuracy and system simplicity compared with conventional high-throughput cytometry systems, which currently suffer from both crowded colours and fluctuating intensities. This result suggests the feasibility of 'digital cytometry' based on lifetime codes, without colour/intensity compensation requirements. The recorded $\tau$-Dots-stained Giardia images, over hours of continuous illumination, show no observable photobleaching effects (Fig. 2c). Moreover, our water-soluble $\tau$-Dots functionalized by different surface groups maintain their lifetime features across different biological application media (Supplementary Section 5) and intracellular environments (Supplementary Section 4.2). Small $\tau$-Dots can also be produced with sizes as small as $\sim 10 \mathrm{~nm}$ (Supplementary Section 1.2). These results indicate that our lifetime-encoded $\tau$-Dots comprise robust labelling materials for bioimaging.

The $\tau$-Dots also relax the multiplexing constraints in suspension arrays, where the ensembles of microspheres are currently limited to only 100 colour- and intensity-coded channels (10 intensity levels at two colour emission bands) ${ }^{28,29}$. We have been able to create a new matrix of lifetime-coded microspheres by simply absorbing the asprepared nanocrystals onto the shell of porous polystyrene microspheres (Fig. 3 and Supplementary Section 6). Both the lifetime and intensity of individual microspheres have been recorded during rapid scanning by our time-resolved scanning cytometry system. As shown in Fig. 4a, the emitter (Tm) variation within the range $0.2-8 \mathrm{~mol} \%$ (with the sensitizer $\mathrm{Yb}$ fixed at $20 \mathrm{~mol} \%$ and a nanocrystal size of $\sim 40 \mathrm{~nm}$ ) resulted in a remarkably large range of lifetimes from $25.6 \mu \mathrm{s}$ to $662.4 \mu \mathrm{s}$ in the blue band. Increasing the $\mathrm{Yb}$ concentration from 10 to $30 \mathrm{~mol} \%$ (for $\mathrm{Tm}$
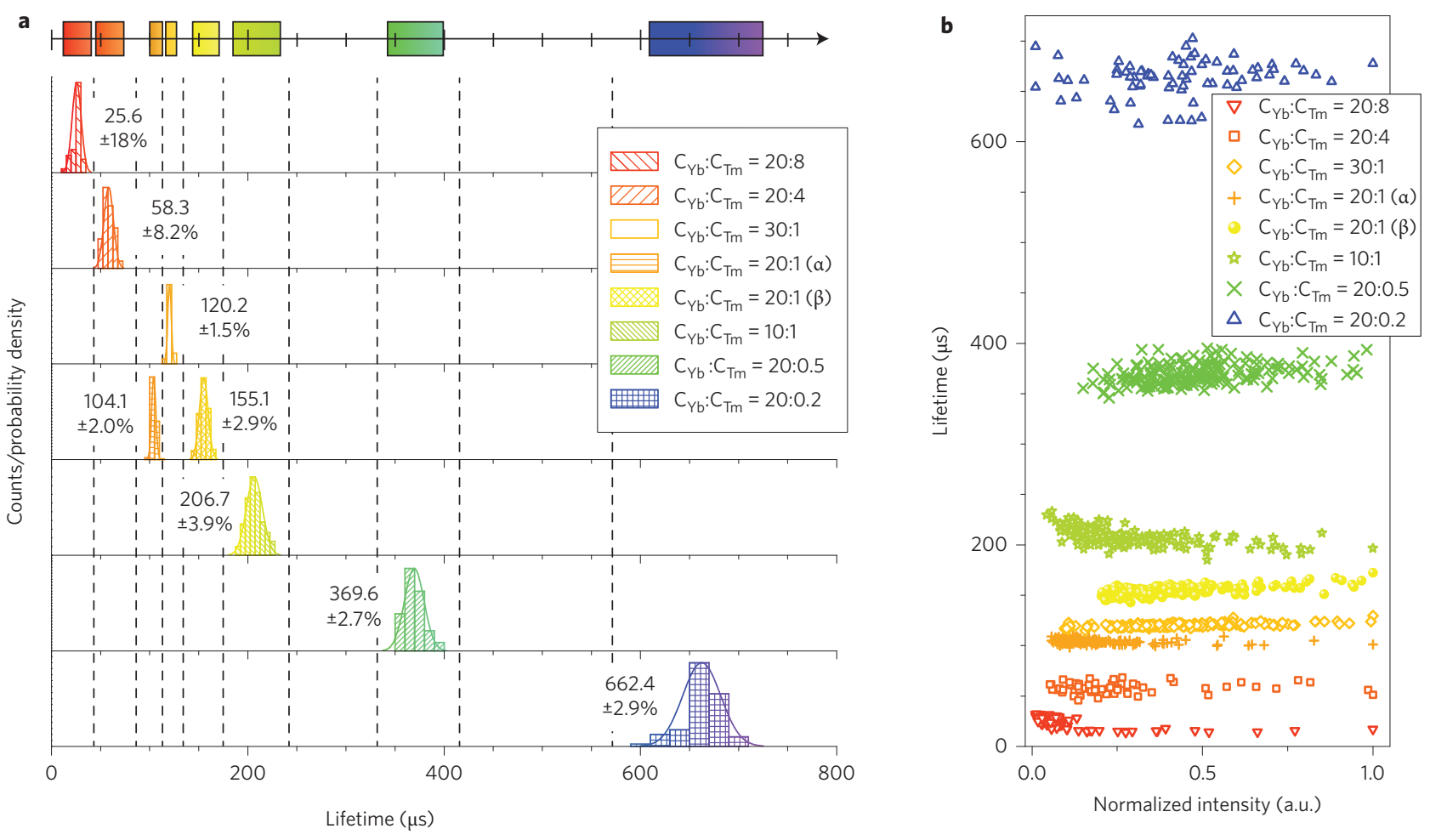

Figure 4 | Results for $\boldsymbol{\tau}$-Dots-encoded populations of microspheres carrying unique lifetime identities. $\mathbf{a}$, The mechanism of upconversion energy transfer, by adjusting the co-dopant concentration of the sensitizer/emitter, can generate eight lifetime populations of microspheres in the Tm blue-emission band. Symbols $\alpha$ and $\beta$ represent cubic and hexagonal crystal phases, respectively. The numeral besides each histogram is the mean lifetime \pm lifetime $C V$ from Gaussian distribution fitting. The blocks in the axis above represent the lifetime resources $( \pm 3 \sigma)$ occupied by each population. The open spaces suggest more populations could be engineered. $\mathbf{b}$, Two-dimensional (intensity versus lifetime) scattered plots showing that all lifetime populations are independent of the intensities of individual microcarriers. 


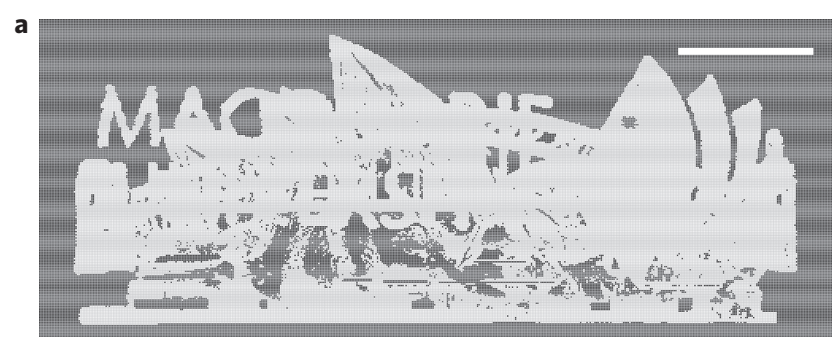

b
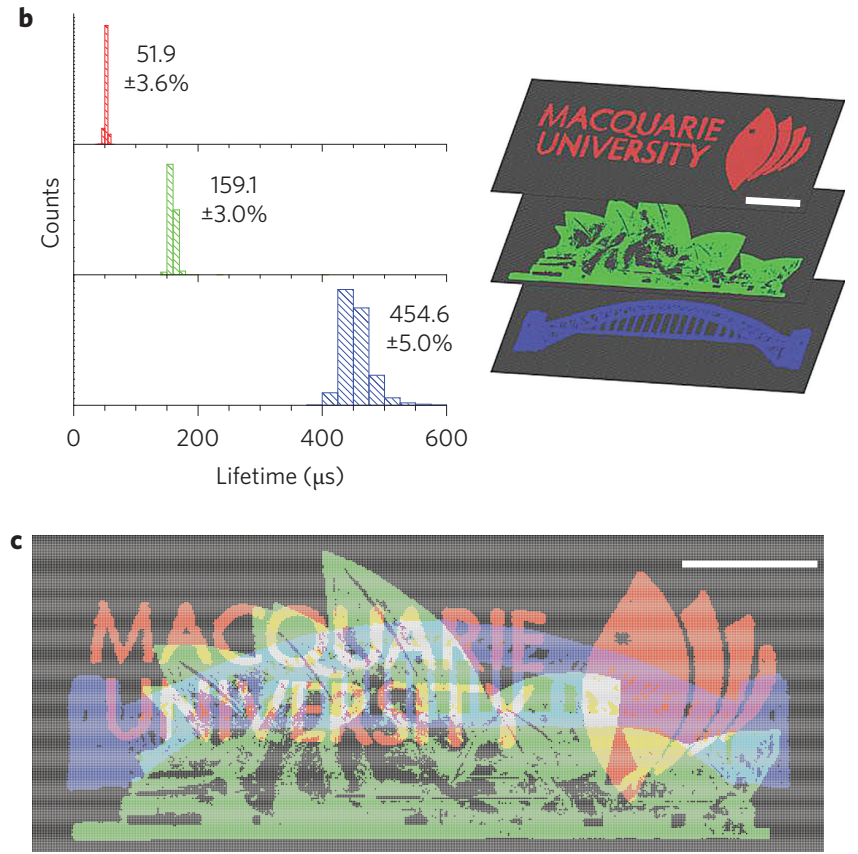

Figure 5 | Demonstration of lifetime-encoded document security and photonic data storage. a-c, Three overlapping patterns are printed with different Tm $\tau$-Dots: ( $C_{Y b}: C_{T m}$ ) 20:4 for the 'Macquarie University' logo, 20:1 for the Sydney Opera House image, and 20:0.5 for the Sydney Harbour Bridge image. Intensity-based luminescence imaging only gives a complex picture (a), but time-resolved scanning separates the patterns based on the lifetime components of every pixel (b), so that genuine multiplexing information contained in the same overlapping space of the document can be decoded (c; pseudocolour is used to indicate the luminescence lifetime for each pixel). Scale bars (all images), $5 \mathrm{~mm}$

fixed at $1 \mathrm{~mol} \%$ and nanocrystal size at $\sim 40 \mathrm{~nm}$ ) also significantly accelerated the blue emission decay from 206.7 to $120.2 \mu \mathrm{s}$. We also found that cubic-phase crystals typically yield shorter lifetimes than the hexagonal phase due to an increased level of crystal defects and internal quenching ${ }^{30,31}$. By using this approach we have been able to tune the lifetime of $20 \mathrm{Yb} / 1 \mathrm{Tm}$ (mol\%/mol\%) nanocrystals from $155.1 \mu \mathrm{s}$ (40 $\mathrm{nm}$ in the hexagonal phase) to $104.1 \mu \mathrm{s}$ (10 $\mathrm{nm}$ in the cubic phase). Interestingly, for each of these population groups, even though the intensity varies across two orders of magnitude (Fig. 4b), the lifetime CV can be as low as $1.5 \%$ (at most $3.9 \%$ for most populations other than the two with the shortest lifetimes, which have slightly larger CVs due to the small average values). These results show that the lifetime codes are independent of intensity, and therefore require no compensation, a further overwhelming advantage over the spectral-domain cytometry method. The narrow CVs helped us realize at least eight completely separate lifetime channels in the blue band, and there is sufficient potential for a total of ten channels or more (note the large gaps among the last three populations). We also synthesized core-shell nanocrystals with further increased lifetime values. For example, by coating our $\tau$-Dots with an inert shell, a thin layer of undoped $\mathrm{NaYF}_{4}$ crystal structure (Supplementary Section 1.4), we significantly extended the typical lifetime of $20 \mathrm{Yb} / 0.5 \mathrm{Tm}$ nanocrystals by $\sim 32 \%$ (Supplementary Table 3 ).

The purpose-engineered multiplexing $\tau$-Dots carrying distinguishable lifetime codes will have further impact in the area of photonics, in particular for high-capacity data storage and data security, in addition to the other optical dimensions currently available such as wavelength and polarization, as well as spatial dimensions. Figure 5 presents a blended picture superimposed by three overlapping images printed with $\tau$-Dots with $\mathrm{Yb}$ :Tm ratios of 20:4, 20:1 and 20:0.5 (mol\%:mol\%), respectively (Supplementary Section 7). Although normal luminescent colour imaging does not show what is concealed in the blended picture (Fig. 5a), our time-resolved scanning cytometry clearly decodes it into three individual images as a result of them having distinct lifetimes of 52, 159 and $455 \mu \mathrm{s}$ (Fig. 5b). This result forms the basis for a new type of document encryption with our $\tau$-Dots as security inks; only authorized individuals who know the correct decoding rule of the luminescence lifetimes are able to access the secret information. Furthermore, a regular arrangement of such nanomaterials into submicrometre-scale units provides a new option for high-density data storage using lifetimes as digits. In particular, the presence/absence of one lifetime component can be used as one binary digit, allowing an 8-bit capacity to be achieved for each unit by simply using three types of lifetime-encoded $\tau$-Dots of the same colour. In either case, one outstanding virtue of our method is that the complexity of the decoding system is not increased, because no additional light sources, filters or detectors are required.

Based on the above demonstrations, we confirmed that the $\tau$-Dots-powered lifetime multiplexing method in the microsecond region has several significant advantages. (1) Multiple physical mechanisms with robust engineering capability can be used to fine-tune and create a series of distinguishable lifetime features without observable crosstalk between different channels (Supplementary Section 8). (2) Multiple emitters in a $\tau$-Dot provide non-bleaching and non-blinking luminescence to stabilize the lifetime feature across different application media, which is ideal for long-term tracking of multiplexed single $\tau$-Dots. (3) Time-resolved detection of exceptionally long luminescence in the microsecond domain completely eliminates any autofluorescence background from usually complex biological samples or embedding substrates. (4) Unlike fluorescence colours, the decoding of the luminescence lifetimes is independent of absolute intensity, so it is more tolerant of ambient background, electronic noise and varying collection efficiencies, as well as possible chromatic aberration associated with optical defocusing. (5) It is compatible with fluorescence lifetime imaging microscopy (FLIM) ${ }^{32,33}$; the amplified bright luminescence from single $\tau$-Dots and objects stained with them enables direct tracking of multiple photons for rapid lifetime measurement (Supplementary Section 2.1), which in the imaging perspective suggests a microsecond-to-millisecond region FLIM powered by our tunable $\tau$-Dots. (6) The upconversion $\tau$-Dots can absorb (stepwise) two or more low-energy photons, thus only requiring low-cost infrared diode excitation, so decoding instrumentation can be as simple as one laser diode and a singleelement photodetector.

In summary, we have successfully demonstrated a new temporaldomain approach to multiplexing. We have introduced a family of nanotags as well as microcarriers with separate lifetime identities independent of colour and intensity. These were decoded without observable crosstalk. This work lays the foundation for future libraries of nano-/microprobes carrying more than 10,000 distinguishable codes (via a combination of colour, intensity and lifetime), unlocking the hidden potential of luminescence as a powerful analytical technique able to cope with the complexity challenges in life sciences and medicine. It also opens new 
opportunities for high-density data storage as well as document security against forgery.

\section{Received 16 May 2013; accepted 31 October 2013; published online XX XX 2013}

\section{References}

1. Zijlstra, P., Chon, J. W. M. \& Gu, M. Five-dimensional optical recording mediated by surface plasmons in gold nanorods. Nature 459, 410-413 (2009).

2. Jeevan, M. M. et al. Security printing of covert quick response codes using upconverting nanoparticle inks. Nanotechnology 23, 395201 (2012).

3. Li, Y. G., Cu, Y. T. H. \& Luo, D. Multiplexed detection of pathogen DNA with DNA-based fluorescence nanobarcodes. Nature Biotechnol. 23, 885-889 (2005).

4. Lin, C. X. et al. Submicrometre geometrically encoded fluorescent barcodes selfassembled from DNA. Nature Chem. 4, 832-839 (2012).

5. Lu, J. et al. MicroRNA expression profiles classify human cancers. Nature $\mathbf{4 3 5}$, 834-838 (2005)

6. Schena, M., Shalon, D., Davis, R. W. \& Brown, P. O. Quantitative monitoring of gene-expression patterns with a complementary-DNA microarray. Science 270, 467-470 (1995)

7. Thomson, J. M., Parker, J., Perou, C. M. \& Hammond, S. M. A custom microarray platform for analysis of microRNA gene expression. Nature Methods 1, 47-53 (2004).

8. Nicewarner-Pena, S. R. et al. Submicrometer metallic barcodes. Science 294 137-141 (2001)

9. Pregibon, D. C., Toner, M. \& Doyle, P. S. Multifunctional encoded particles for high-throughput biomolecule analysis. Science 315, 1393-1396 (2007).

10. Braeckmans, K. et al. Encoding microcarriers by spatial selective photobleaching Nature Mater. 2, 169-173 (2003).

11. Han, M. Y., Gao, X. H., Su, J. Z. \& Nie, S. Quantum-dot-tagged microbeads for multiplexed optical coding of biomolecules. Nature Biotechnol. 19, 631-635 (2001)

12. Wang, F. et al. Tuning upconversion through energy migration in core-shell nanoparticles. Nature Mater. 10, 968-973 (2011).

13. Cunin, F. et al. Biomolecular screening with encoded porous-silicon photonic crystals. Nature Mater. 1, 39-41 (2002).

14. Cao, Y. W. C., Jin, R. C. \& Mirkin, C. A. Nanoparticles with Raman spectroscopic fingerprints for DNA and RNA detection. Science 297, 1536-1540 (2002).

15. Bendall, S. C. et al. Single-cell mass cytometry of differential immune and drug responses across a human hematopoietic continuum. Science 332, 687-696 (2011)

16. Lander, E. S. et al. Initial sequencing and analysis of the human genome. Nature 409, 860-921 (2001).

17. Bartel, D. P. MicroRNAs: genomics, biogenesis, mechanism, and function. Cell 116, 281-297 (2004).

18. Pawson, T. \& Nash, P. Assembly of cell regulatory systems through protein interaction domains. Science 300, 445-452 (2003).

19. Nicholson, J. K. \& Lindon, J. C. Systems biology-metabonomics. Nature $\mathbf{4 5 5}$, 1054-1056 (2008).

20. Van't Veer, L. J. \& Bernards, R. Enabling personalized cancer medicine through analysis of gene-expression patterns. Nature 452, 564-570 (2008).

21. Li, X., Lan, T.-H., Tien, C.-H. \& Gu, M. Three-dimensional orientationunlimited polarization encryption by a single optically configured vectorial beam. Nature Commun. 3, 998 (2012).
22. Perfetto, S. P., Chattopadhyay, P. K. \& Roederer, M. Seventeen-colour flow cytometry: unravelling the immune system. Nature Rev. Immunol. 4, 648-U645 (2004)

23. Cui, H. H., Valdez, J. G., Steinkamp, J. A. \& Crissman, H. A. Fluorescence lifetime-based discrimination and quantification of cellular DNA and RNA with phase-sensitive flow cytometry. Cytometry A 52A, 46-55 (2003).

24. Watson, D. A. et al. A flow cytometer for the measurement of Raman spectra Cytometry A 73A, 119-128 (2008).

25. Gnach, A. \& Bednarkiewicz, A. Lanthanide-doped up-converting nanoparticles: merits and challenges. Nano Today 7, 532-563 (2012).

26. Wang, F. et al. Simultaneous phase and size control of upconversion nanocrystals through lanthanide doping. Nature 463, 1061-1065 (2010).

27. Lu, Y., Xi, P., Piper, J. A., Huo, Y. \& Jin, D. Time-gated orthogonal scanning automated microscopy (OSAM) for high-speed cell detection and analysis. Sci. Rep. 2, 837 (2012)

28. Yang, L., Tran, D. K. \& Wang, X. BADGE, BeadsArray for the detection of gene expression, a high-throughput diagnostic bioassay. Genome Res. 11, 1888-1898 (2001)

29. Yurkovetsky, Z. R. et al. Multiplex analysis of serum cytokines in melanoma patients treated with interferon-alpha 2b. Clin. Cancer Res. 13, 2422-2428 (2007).

30. Wang, F., Wang, J. \& Liu, X. Direct evidence of a surface quenching effect on size-dependent luminescence of upconversion nanoparticles. Angew. Chem. Int Ed. 49, 7456-7460 (2010)

31. Zhao, J. et al. Upconversion luminescence with tunable lifetime in $\mathrm{NaYF}_{4}: \mathrm{Yb}, \mathrm{Er}$ nanocrystals: role of nanocrystal size. Nanoscale 5, 944-952 (2013).

32. Bastiaens, P. I. H. \& Squire, A. Fluorescence lifetime imaging microscopy: spatial resolution of biochemical processes in the cell. Trends Cell Biol. 9, 48-52 (1999).

33. Heilemann, M. et al. High-resolution colocalization of single dye molecules by fluorescence lifetime imaging microscopy. Anal. Chem. 74, 3511-3517 (2002).

\section{Acknowledgements}

The authors thank D. Birch for sample characterization and F. Chi for technical assistance. This project was financially supported by the Australian Research Council (DP 1095465), the China Scholarship Council and Macquarie University Postgraduate Research Scholarships. Y.Lu and D.J. acknowledge the International Society for Advancement of Cytometry for support as ISAC Scholars. P.X. acknowledges support from the ' 973 ' programme of China (2011CB707502, 2011CB809101). D.J. and A.S. acknowledge support through Macquarie University Vice-Chancellor's Innovation Fellowships.

\section{Author contributions}

D.J., J.A.P., R.C.L. and J.P.R. conceived the project. D.J. designed the experiments and supervised the research. Y.Lu, J.Z. and D.J. were primarily responsible for data collection and analysis. Y.Lu, E.M.G. and D.J. prepared figures and wrote the main manuscript text. Y.Lu, J.Z., R.Z., D.L. and D.J. were primarily responsible for the Supplementary Information. All authors contributed to data analysis, discussions and manuscript preparation.

\section{Additional information}

Supplementary information is available in the online version of the paper. Reprints and permissions information is available online at www.nature.com/reprints. Correspondence and requests for materials should be addressed to D.J.

\section{Competing financial interests}

The authors declare no competing financial interests. 